\title{
Empirical Study of Cocyclic Copurity and the Dualization of Cyclic Purity
}

\author{
Md. Arshaduzzaman, PhD \\ M.Sc (Mathematics) \\ Department of Mathematics \\ G.B.College, Naugachia \\ T.M.Bhagalpur University, \\ Bhagalpur, Bihar (India)
}

\author{
Yusuf Perwej, PhD \\ M.Tech(Computer Science \& \\ Engg.) \\ Assistant Professor \\ Department of Computer \\ Information System \\ Al Baha University, Al Baha, \\ Kingdom of Saudi Arabia (KSA)
}

\author{
Ashwani Kumar Sinha, PhD \\ M.Sc (Mathematics) \\ Associate Professor \\ Department of Mathematics \\ M.M. Mahila College \\ Ara, Bihar, India
}

\begin{abstract}
In this paper, we discussed about the Co-cylic co-purity of the dualization of cyclic purity i. e., the Co-purity versus Cohn's purity and the C-purity versus $\mathrm{CP}$ and the Co-cyclic co-purity versus purity and Co-cyclic co-purity versus Cpurity. Many examples are given to show that the concepts of Co-cyclic Co-purity and Cyclic purity are independent.
\end{abstract}

\section{Keywords}

Cyclic Purity, Co-Cyclic Copurity, Cohn's Purity, Projective, Co-Finitly, Polynomial, Ring, R-Module.

\section{INTRODUCTION}

In model theory, the notation of pure, exact sequence is more useful than split exact sequences. There are several variants of this notion. R.Wisbauer [20] generalized the notion of purity for a class $\mathrm{P}$ of /?- modules. He defines a short exact sequence $\quad 0 \rightarrow \mathrm{A} \rightarrow \mathrm{B} \rightarrow \mathrm{C} \rightarrow 0$ of $\mathrm{R}$ modules to be $\mathrm{P}$ pure, if every member of $\mathrm{P}$ is projective with respect to this sequence. Cohn's [3] purity is precisely P-purity for the class $\mathrm{P}$ of all finitely presented R-modules. Cohn's purity is called as purity.

The two more types of P-purity are of interest. One introduced by Simmons [15], called cyclic purity, in which $\mathrm{P}$ is the class of all cyclic P-modules. In the Section 3, we give examples to show that the cyclic purity and Cohn's purity are independent. Another type of P-purity considered by DivaaniAazar'et. al. [4], called Cyclically purity, in which $P$ is the class of R-modules which are isomorphic to $\mathrm{Rn} / \mathrm{G}$ where $\mathrm{n}$ is any natural number and $G$ any cyclic submodule of $\mathrm{Rn}$. Actually, this purity is a generalization of Cohn's purity. Divaani[4], proved that this purity is precisely the intersection purity which was introduced by Stenstrom. In Section 4, we give examples to show that the concepts of cyclic pure and cyclically pure are independent. The First author dualized the Cohn's purity, by introducing Copurity[7]. In this paper, we study the co-cyclic co-purity as the dualization of cyclic purity.

\section{DEFINITIONS AND NOTATIONS}

In this paper, by a ring $\mathrm{R}$ we mean an associative ring with unity and by MM R- module we mean a unitary right .Rmodule.Consider a short exact sequence, $\epsilon: 0 \rightarrow \mathrm{A} \rightarrow \mathrm{B} \rightarrow \mathrm{C}$ $\rightarrow 0$ of $\mathrm{R}$ modules We call an R-module $\mathrm{M}$ to be $\epsilon-\mathrm{i}$ injective (resp. $\epsilon$-projective) if $\mathrm{M}$ is injective (resp. projective) with respect to the short exact sequence $\epsilon$.

\subsection{Definitions}

An exact sequence $\epsilon: 0 \rightarrow \mathrm{A} \rightarrow \mathrm{B} \rightarrow \mathrm{C} \rightarrow 0$ of $\mathrm{R}$ - modules is said to be cyclic pure (c-pure in short) if every cyclic Rmodule is $\epsilon$-projective.

\subsection{Definitions}

An exact sequence $\epsilon: 0 \rightarrow \mathrm{A} \rightarrow \mathrm{B} \rightarrow \mathrm{C} \rightarrow 0$ of R-modules is said to be cyclic pure (CP in short) if every R-module which is isomorphic to $\mathrm{R}^{\mathrm{n}} / \mathrm{G}$ where $\mathrm{n}$ any natural number and $\mathrm{G}$ cyclic submodule of $\mathrm{R}^{\mathrm{n}}$, is $\epsilon$-projective.

\subsection{Definitions}

An R-module $\mathrm{M}$ is said to be co-cyclic, if it can be embedded in the injective hull of a simple R-module. $M$ is said to be sub directly irreducible if the intersection of any family of nonzero submodules of $\mathrm{M}$ is nonzero. It. is easy to prove that the concepts of co-cyclic module and subdirectly irreducible module are equivalent.

\subsection{Definitions}

An It module $M$ in said to be finitely embedded (f.e) [18] (later called by J.P. Jans [8] as co-fmitely generated) if, E(M] $=\mathrm{E}\left(\mathrm{S}_{1}\right) \oplus \mathrm{E}\left(\mathrm{S}_{2}\right) \oplus \ldots . . \oplus \mathrm{E}\left(\mathrm{S}_{\mathrm{n}}\right)$ for some simple $\mathrm{R}$ modules $S_{1}, \ldots, S_{n}$ (here $E(A)$ denotes the injective hull of an R-module A).

\subsection{Definitions}

A R-module $\mathrm{M}$ is said Otis was finitely co-generated [1, p. 124], if the following condition is satisfied. If 3 is any family of submodules of $M$ such that $\cap Z=(0)$ then there is finite subfamily $\mathrm{F}$ of 3 such that $\cap Z=(0)$. It was shown unit the concepts of finite embedded ami finitely co-generated are same CF [8].

\subsection{Definitions}

An R-module M is said to be cofree [6], if $\mathrm{M}$ is embeddable in a direct product, of the injective hulls of a family of simple Rmodules.

\subsection{Definitions}

A R-module $\mathrm{M}$ is co-finitely related if there exists a short exact sequence, $0 \rightarrow \mathrm{M} \rightarrow \mathrm{N} \rightarrow \mathrm{K} \rightarrow 0$ where $\mathrm{N}$ is cofree and $\mathrm{N}$ and $\mathrm{K}$ are co-finitly generated.

\subsection{Definitions}

An exact sequence, $\epsilon: 0 \rightarrow \mathrm{A} \rightarrow \mathrm{B} \rightarrow \mathrm{C} \rightarrow 0$ of $\mathrm{R}$ modules is said to be copure if every co-finitely related R-modules is $\epsilon$ 
-i injective.

\subsection{Definitions}

A submodule A of an R-module B is said to be $\cap$-pure B [16] if $\mathrm{AI}=\mathrm{BI} \cap \mathrm{A}$, for every left ideal $\mathrm{I}$ of $\mathrm{R}$.

\section{C-PURITY VERSUS COHN'S PURITY 3.1 Case: In General Purity Does Not Imply C-Purity}

Let $\mathrm{R}=\prod_{\alpha \in \Lambda} \mathrm{R}_{0}$ and $\mathrm{S}=\oplus_{\alpha \in \mathrm{A}} \mathrm{R}_{0}$ where $\{\mathrm{R} 0\}$ is any infinite family of fields. Clearly $\mathrm{S}$ in an essential ideal of R. If $\mathrm{S}$ is cyclic pure, in $\mathrm{R}$ then $\mathrm{R} / \mathrm{S}$ is projective with respect to the canonical short exact sequence $0 \rightarrow \mathrm{S} \rightarrow \mathrm{R} \rightarrow \mathrm{R} / \mathrm{S} \rightarrow 0$. Then $\mathrm{S}$ is a direct summand of $\mathrm{R}$. Since, $\mathrm{S}$ is an essential ideal of $R$, this implies that $R=S$, which is impossible. So, $S$ is not cyclic pure in $\mathrm{R}$. Since, $\mathrm{R}$ is a von-Neumann regular ring by [17. Proposition 11.1], $\mathrm{S}$ is pure in $\mathrm{R}$.

\subsection{Case: In General Cyclic Purity Does Not Imply Purity}

Let $\mathrm{R}=\mathrm{k}[\mathrm{X}, \mathrm{Y}]$ be a polynomial ring in two variables $\mathrm{X}, \mathrm{Y}$ over a field $\mathrm{k}$. Here, the ideal $(\mathrm{X}, \mathrm{Y})$ of $\mathrm{R}$ is torsion-free, but not flat as an R-module. (( cf. [1.Clmpter I, Exercise 2.3])). Let $(X, Y)=F / K$ for a free module $F$ and a submodule $\mathrm{K}$. Since, $(X, Y)=F / K$ is not flat, $K$ is not pure in $F$. Since, $R$ is a commutative integral domain, by $[15$, Remark $\mathrm{C}], \mathrm{K}$ is c-pure in $\mathrm{F}$.

\section{THE C-PURITY VERSUS CP 4.1 Case: In General CP Docs Not Imply C-Purity}

Since, CP is a generalization of purity, a pure, exact sequence is always CP-exact. Hence, the example given after the Remark 3.1, serves our purpose.

\subsection{Case: In General C-Purity Does Not Imply CP}

Consider the example given after Remark 3.2. In that example, if $\mathrm{K}$ is $\mathrm{CP}$ in F, then by [4, Proposition 2.2], $\mathrm{KI}=\mathrm{K} \cap \mathrm{FI}$, for every ideal I of $\mathrm{R}$. Then by $[20,36.6],(X, Y)$ is flat, which is a contradiction. Hence $\mathrm{K}$ is not a $\mathrm{CP}$ submodule of $\mathrm{F}$.

\section{THE CO-CYCLIC COPURITY}

In this section, we study the co-cyclic co-purity, which is given by Choudhari and Tiwari [2]. But we study the concept as the dualization of c-purity.

\subsection{Definitions}

A short exact sequence, $\epsilon: 0 \rightarrow \mathrm{A} \rightarrow \mathrm{B} \rightarrow \mathrm{C} \rightarrow 0$ of $\mathrm{R}$ modules is said to be co-cyclically copure [2, p.1568] (in short ccp) if every cocyclic R-modulc is $\epsilon$-injective.

\subsection{Definitions}

A submodule A of an R-module B is said to be a ccp submodule of $B$ if the canonical short exact sequence, $\epsilon: 0 \rightarrow$ $\mathrm{A} \rightarrow \mathrm{B} \rightarrow \mathrm{B} / \mathrm{A} \rightarrow 0$ of $\mathrm{R}$-modid fx i.s cc.p. Choudhary and Tiwari [2. Proposition 4.2] have proved the following characterization for ccp submodule.

\subsection{Proposition}

For a submodule A of an R-module B, the following conditions are equivalent. i) $\mathrm{A}$ is ccp in $\mathrm{H}$.

ii) For each $0 \neq a \in A$ and a submodule $C$ of $A$ maximal with respect to the property not containing a, there exists a submodule $\mathrm{D}$ of $\mathrm{B}$ containing $C$ maximal with the following properties $\mathrm{a} \notin \mathrm{D}, \mathrm{C}=\mathrm{D} \cap$ $\mathrm{A}$ and $\mathrm{B}=\mathrm{D}+\mathrm{A}$.

\subsection{Proposition}

Let A, B,C be R-rnodule such that A is a submodule of B and $\mathrm{B}$ is a submodule of $\mathrm{C}$.

i) If $A$ is ccp in B and B is ccp in $C$ then $A$ is ccp in C.

ii) If $A$ is ccp in $C$ then $A$ is ccp in $B$.

iii) If $\mathrm{B}$ is ccp in $\mathrm{C}$ then $\mathrm{B} / \mathrm{A}$ is ccp in $\mathrm{C} / \mathrm{A}$.

iv) If $\mathrm{A}$ is $c c p$ in $\mathrm{C}$ and $\mathrm{B} / \mathrm{Ais}$ ccp in $\mathrm{C} / \mathrm{A}$ then $\mathrm{B}$ is ccp inC.

Proof: The proof solution (i), (ii), (iii) are straightforward., We prove (iv)

Let $\mathrm{M}$ be a co-cyclic R-module and $\mathrm{f} \epsilon \operatorname{Hom}(\mathrm{B}, \mathrm{M})$. Let $\mathrm{f}_{1}$ $=\mathrm{f} / \mathrm{A}$ be the restriction map. Since, $\mathrm{A}$ is ccp in $\mathrm{C}$, there exists $g_{1} \in \operatorname{Hom}(C, M)$ such that $g_{1} / A=f_{1}$. Let $v$. Since $h=f-k$ vanishes on $A$, it induces a homomorphism $h^{+}: B / A \rightarrow M$ defined $\mathrm{f}$ by $\mathrm{h}^{+}(\mathrm{b})=\mathrm{g}_{1}(\mathrm{~b})-\mathrm{f}(\mathrm{b})$. Since $\mathrm{B} / \mathrm{A}$ is ccp in C/A, there exists $\mathrm{g}_{2} \in$ Hom $(\mathrm{C} / \mathrm{A}, \mathrm{M})$ such that $\mathrm{g}_{2} /(\mathrm{B} / \mathrm{A})=\mathrm{h}^{*}$. Now define a map $\mathrm{g}: \mathrm{C} \rightarrow \mathrm{M}$ by $\mathrm{g}^{\prime}(\mathrm{c})=\mathrm{g}_{1}(\mathrm{c})-\left(\mathrm{g}_{2} \mathrm{O} \eta(\mathrm{c})\right)$. Clearly, $g$ is a well defined homomorphism. Now, if $b \in B$, $\mathrm{g}(\mathrm{b})=\mathrm{g}_{1}(\mathrm{~b})-\mathrm{g}_{2}(\mathrm{~b})=\mathrm{g}_{1}(\mathrm{~b})-\mathrm{f}_{2}(\mathrm{~b})=\mathrm{g}_{1}(\mathrm{~b})-\left(\mathrm{g}_{1}(\mathrm{~b})-\mathrm{f}(\mathrm{b})\right)=\mathrm{f}$ (b) . Hence, $g / B=f$. Hence, the result.

\subsection{Case}

By the Proposition 5.4, it is clear that the family of all ccp short exact sequences of R-rnodule forms a Proper Class in the sense of MacLane [10].

\subsection{Proposition}

i) If $\mathrm{A}$ is a co-cyclic, ccp submodule of an R-module B then $\mathrm{A}$ is a direct summand of $\mathrm{B}$.

Proof : Since, by hypothesis, A is co-cyclic, ccp submodule of B the identity map IA of A extends to an R-homomorphism from $\mathrm{B}$ to $\mathrm{A}$. This implies that $\mathrm{A}$ is a direct siimmand of $\mathrm{B}$.

ii) A submodule $\mathrm{B}$ of an R-module $\mathrm{C}$ is ccp in $\mathrm{C}$ if and only if for every sub module A of B such that B/A is co-cyclic, B/A is a direct summand of $\mathrm{C} / \mathrm{A}$.

Proof: Only if the let A be a submodule of B such that B/A is co-cyclic. Since, by hypothesis, B is ccp in C, it follows by Proposition 5.4 (iii), that $\mathrm{B} / \mathrm{A}$ is ccp in $\mathrm{C} / \mathrm{A}$. It now follows by (i) above that $\mathrm{B} / \mathrm{A}$ is a direct summand of $\mathrm{C} / \mathrm{A}$.

If: Let $\mathrm{f}: \mathrm{B} \rightarrow \mathrm{M}$ be any $\mathrm{R}$-homomorphism from $\mathrm{B}$ into a cocyclic R-module $M$. Let $A=\operatorname{Ker} f$. Then $f$ induces a monomorphism $\mathrm{f}^{*}: \mathrm{B} / \mathrm{A} \rightarrow \mathrm{M}$ from $\mathrm{B} / \mathrm{A}$ into $\mathrm{M}$. Since, $\mathrm{M}$ is cocyclic so is $\mathrm{B} / \mathrm{A}$. Then, by hypothesis, $\mathrm{B} / \mathrm{A}$ is a direct summand of C/A. So, there exists an R-homomorphism $\theta$ : C/ $\mathrm{A} \rightarrow \mathrm{B} / \mathrm{A}$ which is identity on $\mathrm{B} / \mathrm{A}$. Let $\eta: \mathrm{C} \rightarrow \mathrm{C} / \mathrm{A}$ be the canonical epimorphism and let $\mathrm{g}=\mathrm{f}^{*}$ o $\theta$ o $\eta: \mathrm{C} \rightarrow \mathrm{M}$. We prove that $g$ extends $f$. For $b \in B$, we have $g^{\prime}(b)=\left(f^{*}\right.$ o $\theta$ o $\eta)(b)=\left(f^{*}(\theta(\eta))\right)=\left(f^{*}(\theta(b+A))=f^{*}(b+A)=f(b)\right.$. Thus $\mathrm{g} s$ extends $\mathrm{f}$. This proves that $\mathrm{B}$ is a ccp submodule of C. 


\subsection{Proposition}

For a ring $\mathrm{R}$ the following conditions are equivalent.

i) $\mathrm{R}$ is right $\mathrm{V}$-ring.

ii) Every short exact sequence of R-modules is copure.

iii) Every short exact sequence of R-modules is ccp.

iv) Every right ideal of $\mathrm{R}$ rs a ccp submodule of $\mathrm{R}$.

Proof: (i) $\Rightarrow$ (ii): Follows by (i) $\Rightarrow$ (iii) of [7, proposition 5] (ii) $\Rightarrow$ (iii): Follows by the remark after the proof of the Proposition 5 of [7] (iii) $\Rightarrow$ (iv): obvious. (iv) $\Rightarrow$ (i): By [7, Proposition 4(i)], we need only prove that every co-cyclic Rmodule is injective. Let $\mathrm{M}$ be any co-cyclic $\mathrm{R}$-module and let $\mathrm{f}: \mathrm{I} \rightarrow \mathrm{M}$ be any R-homomorphism from a right ideal $\mathrm{I}$ of $\mathrm{R}$ into M. Since, by hypothesis (iv), I is ccp in R, $\mathrm{f}$ extends to an $\mathrm{R}$-homomorphism from $\mathrm{R}$ into $\mathrm{M}$. This proves the in-jectivity of $\mathrm{M}$ by the Baer's criterion of in-jectivity. This proves that $\mathrm{R}$ is a right $\mathrm{V}$-ring.

\subsection{Proposition}

If $\mathrm{A}$ is a, ccp submodule of an $\mathrm{R}$-module $\mathrm{B}$ then, $\mathrm{AI}=\mathrm{BI} \cap$ A, for every right ideal I of $\mathrm{R}$.

Proof: Let $\mathrm{f}$ be any right ideal of R. Clearly AI $\subseteq$ BI $\cap$ A. For the reverse inclusion. let, $x \in B I \cap A$. Suppose $x \notin A I$. Let $\mathrm{K}$ be a submodule of A maximal with the property that AI $\subseteq \mathrm{K}$ and $\mathrm{x} \notin \mathrm{K}$ (we note that since $\mathrm{I}$ is a right ideal of $\mathrm{R}, \mathrm{AI}$ is a submodule of $\mathrm{A}$ ). Then $\mathrm{A} / \mathrm{K}$ is sub directly irreducible ami hence co-cyclic. Let $\eta: A \rightarrow A / K^{\prime}$ be the canonical equimorphism. Since, by hypothesis, A is ccp submodule of B there exists an R-homomorphism $\mathrm{f}: \mathrm{B} \rightarrow \mathrm{A} / \mathrm{K}$ such that $\mathrm{f} / \mathrm{A}=$ $\eta$. Since $\mathrm{x} \in \mathrm{A}, \eta(\mathrm{x})=\mathrm{f}(\mathrm{x}) \in \mathrm{f}(\mathrm{BI}) \subseteq \mathrm{f}(\mathrm{B}) \mathrm{I} \subseteq(\mathrm{A} / \mathrm{K}) \mathrm{I}=($ $\delta$ ) (because $\mathrm{AI} \subseteq \mathrm{K}$ ) which implies that $\eta(\mathrm{x})=\delta$ which is impossible since $\mathrm{x} \notin \mathrm{K}$. Hence $\mathrm{x} \in \mathrm{AI}$. This proves that BI $\cap$ $\mathrm{A} \subseteq \mathrm{AI}$. This completes the proof of the proposition.

\subsection{Corollary}

If a right ideal I is ccp submodule of $\mathrm{R}$, then I is idempotent.

\subsection{Corollary}

If $\mathrm{R}$ is a commulative ring then every ccp submodule of an $\mathrm{R}$ module $\mathrm{M}$ is an $\cap$-pure submodule of $\mathrm{M}$.

\subsection{Corollary}

If $\mathrm{R}$ is a commulative ring and if $0 \rightarrow \mathrm{A} \rightarrow \mathrm{F} \rightarrow \mathrm{B} \rightarrow 0$ be cheap with $\mathrm{F}$ flat, then $\mathrm{B}$ is flat.

Proof: Let $0 \rightarrow \mathrm{A} \rightarrow \mathrm{F} \rightarrow \mathrm{B} \rightarrow 0$ be ccp then by the above proposition $\mathrm{AI}=\mathrm{FI} \cap \mathrm{A}$ for every ideal $\mathrm{I}$ of R. Hence, by [20, 36.6] B is flat. Fuches in [5, p. 121, Ex. 1] has mentioned an equivalent condition fo the pure subgroup. This condition is partially generalized in the following proposition for ccp submodules over comulative rings.

\subsection{Corollary}

If $\mathrm{R}$ is a commulative ring and $\mathrm{A}$ is a ccp submodule of an $\mathrm{R}$ module $\mathrm{B}$ then, $(\mathrm{A}: \mathrm{r})=\mathrm{A}+(0: \mathrm{r})$ where, for an $\mathrm{R}$-submodule $\mathrm{C}$ of $\mathrm{B},(\mathrm{C}: \mathrm{r})=\{\mathrm{b} \in \mathrm{R} / \mathrm{br} \in \mathrm{C}\}$.

Proof: A $\mathrm{A}+(0: \mathrm{r}) \subseteq(\mathrm{A}: \mathrm{r})$ is obvioius. Let $\mathrm{x} \in(\mathrm{A}: \mathrm{r})$ then $\mathrm{xr} \in \mathrm{A}$. Consider the ideal $\mathrm{rR}=\mathrm{I}$ of $\mathrm{R}$. Now $\mathrm{xr} \in \mathrm{BI}$. By above Proposition 5.8, $x r \in$ AI . So, $x r=\sum_{i=1}^{n} a_{i} r_{i}$ for some $a_{i}$ $\epsilon \mathrm{A}$ and $\mathrm{r}_{\mathrm{i}} \in \mathrm{R}$ for $1 \leq \mathrm{i} \leq \mathrm{n}$. Then $\mathrm{xr}=\mathrm{a}_{0} \mathrm{r}$ where, $\mathrm{a}_{0}=$ $\sum_{i=1}^{n} \mathrm{a}_{\mathrm{i}} \mathrm{r}_{\mathrm{i}} \in \mathrm{A}$. Then $\mathrm{xr}-\mathrm{a}_{0} \mathrm{r}=0 \Rightarrow\left(\mathrm{x}-\mathrm{a}_{0}\right) \mathrm{r}=\mathrm{a} \Rightarrow \mathrm{x}-\mathrm{a}_{0}$ $\epsilon(0: r) \Rightarrow x \in A+(0: r)$. This implies, $(\mathrm{A}: \mathrm{r}) \subseteq \mathrm{A}+(0:$ r). Hence the proof.

\section{CCP VERSUS PURITY}

\subsection{Case: In General A Pure Submodule Of A R-Module Need Not Be Ccp Sub- Module}

In, the first author gave an example for a pure submodule which is not co-pure. We prove that the same example serves our purpose.

Let $\mathrm{V}$ be a comfortably infinite dimensional vector space over the field $\mathrm{Q}$ of rational numbers and $\mathrm{R}$ be the ring of linear operators of $\mathrm{V}$. Then $\mathrm{R}$ is von Neumann regular ring by [11, Theorem 7.3]. Let I be the two sided ideal of $f$ ? of all linear operators of $\mathrm{V}$ of finite rank. Then, by [11, Theorem 7.4], I is the only proper ideal of R. Then, by [14, Theorem 1], (0) is the only cyclic, injertivr module annihilated by the maximal two-sided ideal I of R. Let Jbe a maximal right ideal of $\mathrm{R}$ containing I. Since, I annihilates the simple R-module $\mathrm{S}=\mathrm{R} / \mathrm{J}$ this implies $S$ is non-injective R-module and hence $S \neq E(S)$. Let, $0 \neq \mathrm{x} \in \mathrm{E}(\mathrm{S})$ - $\mathrm{S}$. Let $\mathrm{T}$ be a submodule of $\mathrm{E}(\mathrm{S})$ maximal with respect to the property that $\mathrm{S} \subseteq \mathrm{T}$ and $\mathrm{x} \notin \mathrm{T}$. Then, $\mathrm{T}$ is a co-cyclic, essential submodule of $\mathrm{E}(\mathrm{S})$ and $\mathrm{T} \neq \mathrm{E}(\mathrm{S})$. Hence, $T$ cannot be a. direct summand of $H(S)$. By Proposition 5.5(i), $T$ is not a ccp submodule of $E(S)$. Since, $R$ is a von Neumann regular ring, it implies, by [17, Propositions $11.1]$, that every short exact sequence of R-modules is pure. In particular $\mathrm{T}$ is a pure submodule of $\mathrm{E}(\mathrm{S})$.

\subsection{Proposition}

Oner a commulantive Noetherian (co-Noetherian) ring a pure submodule of an R-module is ccp submodule.

Proof: Follows from [7, Proposition 12] and Proposition 5.15.

\section{CCP VERSUS C- PURITY}

\subsection{Remark: In General Ccp Does Not Imply Cyclic Purity}

In the above example, after Remark 3.1 , the ring $\mathrm{R}$ is a commutative von-Neumann regular ring and hence $\mathrm{V}$ ring. So, by Proposition 5.7, $\mathrm{S}$ is ccp in R. But it is proved that $\mathrm{S}$ is not cyclic pure in $\mathrm{R}$.

\subsection{Case: In General Cyclic Purity Does Not Imply Ccp}

In the above example, after Remark $3.2, \mathrm{~K}$ is proved to be cyclic pure in $\mathrm{F}$. But by the Corollary 5.11 above, $\mathrm{K}$ is not ccp in $\mathrm{F}$, since $\mathrm{F} / \mathrm{K}=(\mathrm{X}, \mathrm{Y})$ is not flat.

\section{CONCLUION}

From the above section and examples it is clear that the genral purity does not imply C-purity and in genral cyclic purity does not imply purity and that we get the conclusion that the cocyclic co-purity does not imply cyclic purity and cyclic purity does not imply co-cyclic co-purity.Hence the concepts of cocyclic copurity and cyclic purity are independent.

\section{REFERENCES}

[1] F. W. Anderson and K. R. Fuller, Rings and Categories 
of Modules, Springer-Verlag New York. Heidelberg. Berlin,(1991).

[2] D. P. Choudhary and K. Tewari, Tensor purities, cyclic quasiprojective and Co-cyclic copurity, Gomm. in Alg.,7(1979), 1559-1572.

[3] P. M. Conn, On the free product of associative ringsI.Math.Z.,71(1959),380-398.

[4] K. Divani-Aazar, M. A. Esmkhani and M.Tousi, Some criteria of cyclically pure injective modules, Journal of Algebra., 304(2006), 367-381.

[5] L. Fuchs, Infinite Abelian Groups, Academic Press New York \& London.(1970)

[6] V. A. Hiremath, Cofinitcly generated and cofinitely related modules, Acta.Math. Acad.Sci.Hungar.,39(1982),1-9. Study of Cocyclic Copurity and the Dualization of Cyclic Purity : Ashwani Kumar Sinha

[7] V. A. Hiremath, Hopure submodules, Acta.Math.Hungar.,44(1984), 3-12.

[8] J. P. Jans.On co-Noetherian rings. J.London Math. Soc.(2)1 (1969), 588-590.

[9] T. Y. Lam, A First Course in Noncommutative Rings, Springer publications (1990).
[10] S. S. Mat-Lane, Ilomology, Springer(1967).

[11] N. H. McCoy, The Theory of Rings.The Macmillan company., New York,(1967).

[12] M. S. Osborne, Basic Homological Algebra., SpringerVerlag New York, Inc (2000).

[13] K L. Osofisky. A Generalization of a Quasi- Frohenius rings, Journal of Algebras, 4 (1966), 373-387.

[14] B. L. Osofsky, Cyclic injective modules of full linear rings, Proc.Amer..Math.Soc. 17(1966), 247-253.

[15] J. Simmons,Cyclic purity, a generalization of purity for modules. Houston J.Math. 13, No.l (1987), 135-150.

[16] B. Stenstrom, Pure Submodules, Arkiv for Mat. 7(1967), 159-171.

[17] B. Stenstrom,Rings of Quotients, Springer- Verlag, New York, Heidelberg, Berlin (1975).

[18] P. Vamos, On the dual of the notion of finitely generated, J.London Math.Soc., 43 (1968), 643-646.

[19] R. B. Warfield Jr., Purity and algebraic compactness for modules, Pacific J.Math. 28(3) (1969), 699-719.

[20] R. Wisbauer, Foundations of Modules and Ring Theory, Gordon and Breach Science Publishers (2007). 